Article

\title{
Generalized Hyers-Ulam Stability of the Additive Functional Equation
}

\author{
Yang-Hi Lee ${ }^{1}(\mathbb{D})$ and Gwang Hui Kim ${ }^{2, *}$ (D) \\ 1 Department of Mathematics Education, Gongju National University of Education, Gongju 32553, Korea; \\ yanghi2@hanmail.net \\ 2 Department of Mathematics, Kangnam University, Yongin 16979, Korea \\ * Correspondence: ghkim@kangnam.ac.kr
}

Received: 2 May 2019; Accepted: 21 June 2019; Published: 25 June 2019

\begin{abstract}
We will prove the generalized Hyers-Ulam stability and the hyperstability of the additive functional equation $f\left(x_{1}+y_{1}, x_{2}+y_{2}, \ldots, x_{n}+y_{n}\right)=f\left(x_{1}, x_{2}, \ldots, x_{n}\right)+f\left(y_{1}, y_{2}, \ldots, y_{n}\right)$. By restricting the domain of a mapping $f$ that satisfies the inequality condition used in the assumption part of the stability theorem, we partially generalize the results of the stability theorems of the additive function equations.
\end{abstract}

Keywords: additive (Cauchy) equation; additive mapping; Hyers-Ulam stability; generalized Hyers-Ulam stability; hyperstability

MSC: 39B82; 39B5

\section{Introduction}

In 1940, Ulam [1] gave the question concerning the stability of homomorphisms in a conference of the mathematics club of the University of Wisconsin as follows:

Let $(G, \cdot)$ be a group, and let $\left(G^{\prime}, \cdot, d\right)$ be a metric group with the metric $d$. Given $\delta>0$, does there exist $\epsilon>0$ such that if a mapping $h: G \rightarrow G^{\prime}$ satisfies the inequality

$$
d(h(x y), h(x) h(y)) \leq \delta
$$

for all $x, y \in G$, then there is a homomorphism $H: G \rightarrow H$ with

$$
d(h(x), H(x)) \leq \epsilon
$$

for all $x \in G$ ?

Next year, the Ulam's conjecture was partially solved by Hyers [2] for the additive functional equation.

Theorem 1. [2], Let $X$ and $Y$ be Banach spaces. Suppose that the mapping $f: X \rightarrow Y$ satisfies the inequality

$$
\|f(x+y)-f(x)-f(y)\| \leq \varepsilon, \quad \forall x, y \in X, \quad \varepsilon \text { : constant }
$$

Then, there exists a unique additive mapping

$$
A(x+y)=A(x)+A(y)
$$

such that $\|f(x)-A(x)\| \leq \varepsilon$, where the limit $A(x)=\lim _{n \rightarrow \infty} 2^{-n} f\left(2^{n} x\right)$. 
Thereafter, this phenomenon has been called the Hyers-Ulam stability.

Theorem 2. Let $X$ and $Y$ be Banach spaces. Suppose that the mapping $f: X \rightarrow Y$ satisfies the inequality

$$
\|f(x+y)-f(x)-f(y)\| \leq \theta\left(\|x\|^{p}+\|y\|^{p}\right)
$$

for all $x, y \in X \backslash\{0\}$, where $\theta$ and $p$ are constants with $\theta>0$ and $p \neq 1$. Then, there exists a unique additive mapping $T: X \rightarrow Y$ such that

$$
\|f(x)-T(x)\| \leq \frac{\theta}{\left|1-2^{p-1}\right|}\|x\|^{p}
$$

for all $x \in X \backslash\{0\}$.

Theorem 2 is due to Aoki [3] and Rassias [4] for $0<p<1$, Gajda [5] for $p>1$, Hyers [2] for $p=0$, and Rassias [6] for $p<0$.

In 1994, Găvruta [7] generalized these results for additive mapping by replacing $\theta\left(\|x\|^{p}+\|y\|^{p}\right)$ in (1) by a general function $\varphi(x, y)$, which is called the 'generalized Hyers-Ulam stability' in this paper.

In 2001, the term hyperstability was used for the first time probably by G. Maksa and Z. Páles in [8]. However, in 1949, it seems to have created by D. G. Bourgin [9] that the first hyperstability result concerned the ring homomorphisms.

We say that a functional equation $\mathfrak{D}(f)=0$ is hyperstable if any function $f$ satisfying the equation $\mathfrak{D}(f)=0$ approximately is a true solution of $\mathfrak{D}(f)=0$, which is a phenomenon called hyperstability.

The hyperstability results for the additive (Cauchy) equation were investigated by Brzdęk $[10,11]$.

In this paper, let $V$ and $W$ be vector spaces, $X$ be a real normed space, and $Y$ be a real Banach space. We denote the set of natural numbers by $\mathbb{N}$ and the set of real numbers by $\mathbb{R}$.

For a given mapping $f: V^{n} \rightarrow W$, where $V^{n}$ denotes $V \times V \times \cdots \times V$, let us consider the additive functional equation

$$
f\left(x_{1}+y_{1}, x_{2}+y_{2}, \ldots, x_{n}+y_{n}\right)=f\left(x_{1}, x_{2}, \ldots, x_{n}\right)+f\left(y_{1}, y_{2}, \ldots, y_{n}\right),
$$

for all $x_{i}, y_{i} \in V(i=1,2, \ldots, n)$.

Each solution of the additive functional Equation (3) is called an $n$-variable additive mapping. A typical example for the solutions of Equation (3) is the mapping $f: \mathbb{R}^{n} \rightarrow \mathbb{R}^{l}$ given by $f\left(x_{1}, x_{2}, \ldots, x_{n}\right)=\left(\sum_{i=1}^{n} a_{1 i} x_{i}, \sum_{i=1}^{n} a_{2 i} x_{i}, \ldots, \sum_{i=1}^{n} a_{l i} x_{i}\right)$ with real constants $a_{i j}$.

In this paper, we will prove the generalized Hyers-Ulam stability of the additive functional Equation (3) in the spirit of Găvruta [7], and the hyperstability of the additive functional Equation (3).

\section{Main Results}

For a given mapping $f: V^{n} \rightarrow W$, we use the following abbreviation:

$$
\begin{aligned}
D f\left(x_{1}, y_{1}, x_{2}, y_{2}, \ldots, x_{n}, y_{n}\right):= & f\left(x_{1}+y_{1}, x_{2}+y_{2}, \ldots, x_{n}+y_{n}\right) \\
& -f\left(x_{1}, x_{2}, \ldots, x_{n}\right)-f\left(y_{1}, y_{2}, \ldots, y_{n}\right)
\end{aligned}
$$

for all $x_{1}, y_{1}, x_{2}, y_{2}, \ldots, x_{n}, y_{n} \in V$. We need the following lemma to prove main theorems.

Lemma 1. If a mapping $f: V^{n} \rightarrow W$ satisfies (3) for all $x_{1}, y_{1}, x_{2}, y_{2}, \ldots, x_{n}, y_{n} \in V \backslash\{0\}$, then $f$ satisfies (3) for all $x_{1}, y_{1}, x_{2}, y_{2}, \ldots, x_{n}, y_{n} \in V$. 
Proof. Let $x \in V \backslash\{0\}$ be a fixed element, and let $i \in\{1,2, \ldots, n\}$. For given $x_{i}, y_{i} \in V$, let $x_{i}^{(1)}, x_{i}^{(2)}$, $y_{i}^{(1)}, y_{i}^{(2)}$ be

$$
\begin{array}{ll}
x_{i}^{(1)}=x, x_{i}^{(2)}=-x, y_{i}^{(1)}=x, y_{i}^{(2)}=-x & \text { if } x_{i}=0 \text { and } y_{i}=0, \\
x_{i}^{(1)}=y_{i}, x_{i}^{(2)}=-y_{i}, y_{i}^{(1)}=\frac{y_{i}}{2}, y_{i}^{(2)}=\frac{y_{i}}{2} & \text { if } x_{i}=0 \text { and } y_{i} \neq 0, \\
x_{i}^{(1)}=\frac{x_{i}}{2}, x_{i}^{(2)}=\frac{x_{i}}{2}, y_{i}^{(1)}=x_{i}, y_{i}^{(2)}=-x_{i} & \text { if } x_{i} \neq 0 \text { and } y_{i}=0, \\
x_{i}^{(1)}=\frac{x_{i}}{2}, x_{i}^{(2)}=\frac{x_{i}}{2}, y_{i}^{(1)}=(k+1) y_{i}, y_{i}^{(2)}=-k y_{i} & \text { if } x_{i} \neq 0 \text { and } y_{i} \neq 0,
\end{array}
$$

where $k$ is a fixed integer, such that $\frac{x_{i}}{2}+(k+1) y_{i} \neq 0, \frac{x_{i}}{2}-k y_{i} \neq 0$. Then, $x_{i}^{(1)}, x_{i}^{(2)}, y_{i}^{(1)}, y_{i}^{(2)}, x_{i}^{(1)}+$ $y_{i}^{(1)}, x_{i}^{(2)}+y_{i}^{(2)} \in V \backslash\{0\}$ and $x_{i}^{(1)}+y_{i}^{(1)}+x_{i}^{(2)}+y_{i}^{(2)}=x_{i}+y_{i}$ for all $i=1,2, \ldots, n$.

Hence, the equalities $D f\left(x_{1}^{(1)}, y_{1}^{(1)}, \ldots, x_{n}^{(1)}, y_{n}^{(1)}\right)=0, D f\left(x_{1}^{(2)}, y_{1}^{(2)}, \ldots, x_{n}^{(2)}, y_{n}^{(2)}\right)=0$, $D f\left(x_{1}^{(1)}, x_{1}^{(2)}, x_{2}^{(1)}, x_{2}^{(2)}, \ldots, x_{n}^{(1)}, x_{n}^{(2)}\right)=0$, and $D f\left(y_{1}^{(1)}, y_{1}^{(2)}, y_{2}^{(1)}, y_{2}^{(2)}, \ldots, y_{n}^{(1)}, y_{n}^{(2)}\right)=0$ hold for all $x_{1}, y_{1}, x_{2}, y_{2}, \ldots, x_{n}, y_{n} \in V$. Since the equality

$$
\begin{aligned}
& D f\left(x_{1}, y_{1}, x_{2}, y_{2}, \ldots, x_{n}, y_{n}\right) \\
&= D f\left(x_{1}^{(1)}+y_{1}^{(1)}, x_{1}^{(2)}+y_{1}^{(2)}, x_{2}^{(1)}+y_{2}^{(1)}, x_{2}^{(2)}+y_{2}^{(2)}, \ldots, x_{n}^{(1)}+y_{n}^{(1)}, x_{n}^{(2)}+y_{n}^{(2)}\right) \\
&+D f\left(x_{1}^{(1)}, y_{1}^{(1)}, x_{2}^{(1)}, y_{2}^{(1)}, \ldots, x_{n}^{(1)}, y_{n}^{(1)}\right)+D f\left(x_{1}^{(2)}, y_{1}^{(2)}, x_{2}^{(2)}, y_{2}^{(2)}, \ldots, x_{n}^{(2)}, y_{n}^{(2)}\right) \\
&-D f\left(x_{1}^{(1)}, x_{1}^{(2)}, x_{2}^{(1)}, x_{2}^{(2)}, \ldots, x_{n}^{(1)}, x_{n}^{(2)}\right)-D f\left(y_{1}^{(1)}, y_{1}^{(2)}, y_{2}^{(1)}, y_{2}^{(2)}, \ldots, y_{n}^{(1)}, y_{n}^{(2)}\right)
\end{aligned}
$$

holds for all $x_{1}, y_{1}, x_{2}, y_{2}, \ldots, x_{n}, y_{n} \in V$, we conclude that $f$ satisfies $D f\left(x_{1}, y_{1}, \ldots, x_{n}, y_{n}\right)=0$ for all $x_{1}, y_{1}, x_{2}, y_{2}, \ldots, x_{n}, y_{n} \in V$.

Thereafter, let $i \in\{1,2,3, \ldots, n\}$. For a given element $\left(x_{1}, x_{2}, \ldots, x_{n}\right) \neq(0,0, \ldots, 0)$, we can choose a fixed element $x^{\prime} \neq 0$, such that $x^{\prime} \in\left\{x_{1}, x_{2}, \ldots, x_{n}\right\}$. Moreover, let $x_{i}^{(1)}, x_{i}^{(2)} \in V \backslash\{0\}$ be the elements defined by

$$
\begin{array}{ll}
x_{i}^{(1)}=x_{i}, x_{i}^{(2)}=x_{i} & \text { if } x_{i} \neq 0, \\
x_{i}^{(1)}=x^{\prime}, x_{i}^{(2)}=-x^{\prime} & \text { if } x_{i}=0 .
\end{array}
$$

By using Lemma 1, we can prove the following set of stability theorems.

Theorem 3. Suppose that $f: V^{n} \rightarrow Y$ is a mapping for which there exists a function $\varphi:(V \backslash\{0\})^{2 n} \rightarrow[0, \infty)$, such that

$$
\sum_{m=0}^{\infty} \frac{\varphi\left(2^{m} x_{1}, 2^{m} y_{1}, 2^{m} x_{2}, 2^{m} y_{2}, \ldots, 2^{m} x_{n}, 2^{m} y_{n}\right)}{2^{m}}<\infty
$$

and

$$
\left\|D f\left(x_{1}, y_{1}, x_{2}, y_{2}, \ldots, x_{n}, y_{n}\right)\right\| \leq \varphi\left(x_{1}, y_{1}, x_{2}, y_{2}, \ldots, x_{n}, y_{n}\right)
$$

for all $x_{1}, y_{1}, x_{2}, y_{2}, \ldots, x_{n}, y_{n} \in V \backslash\{0\}$. Then, there exists a unique mapping $F: V^{n} \rightarrow Y$ that satisfies

$$
D F\left(x_{1}, y_{1}, x_{2}, y_{2}, \ldots, x_{n}, y_{n}\right)=0
$$


for all $x_{1}, y_{1}, x_{2}, y_{2}, \ldots, x_{n}, y_{n} \in V$ and

$$
\left\|f\left(x_{1}, x_{2}, \ldots, x_{n}\right)-F\left(x_{1}, x_{2}, \ldots, x_{n}\right)\right\| \leq \sum_{m=0}^{\infty} \frac{\mu\left(2^{m} x_{1}, 2^{m} x_{2}, \ldots, 2^{m} x_{n}\right)}{2^{m+1}}
$$

for all $\left(x_{1}, x_{2}, \ldots, x_{n}\right) \in V^{n} \backslash\{(0,0, \ldots, 0)\}$, where the function $\mu: V^{n} \rightarrow \mathbb{R}$ is defined by

$$
\begin{aligned}
\mu & \left(x_{1}, x_{2}, \ldots, x_{n}\right) \\
:= & \varphi\left(x_{1}^{(1)}, x_{1}^{(2)}, x_{2}^{(1)}, x_{2}^{(2)}, \ldots, x_{n}^{(1)}, x_{n}^{(2)}\right)+2 \varphi\left(\frac{x_{1}^{(1)}}{2}, \frac{x_{1}^{(2)}}{2}, \frac{x_{2}^{(1)}}{2}, \frac{x_{2}^{(2)}}{2}, \ldots, \frac{x_{n}^{(1)}}{2}, \frac{x_{n}^{(2)}}{2}\right) \\
& +\varphi\left(\frac{x_{1}^{(1)}}{2}, \frac{x_{1}^{(1)}}{2}, \frac{x_{2}^{(1)}}{2}, \frac{x_{2}^{(1)}}{2}, \ldots, \frac{x_{n}^{(1)}}{2}, \frac{x_{n}^{(1)}}{2}\right)+\varphi\left(\frac{x_{1}^{(2)}}{2}, \frac{x_{1}^{(2)}}{2}, \frac{x_{2}^{(2)}}{2}, \frac{x_{2}^{(2)}}{2}, \ldots, \frac{x_{n}^{(2)}}{2}, \frac{x_{n}^{(2)}}{2}\right)
\end{aligned}
$$

for all $\left(x_{1}, x_{2}, \ldots, x_{n}\right) \in V^{n} \backslash\{(0,0, \ldots, 0)\}$.

Proof. From the inequality (6) and the equalities

$$
\begin{aligned}
& f\left(2 x_{1}, 2 x_{2}, \ldots, 2 x_{n}\right)-2 f\left(x_{1}, x_{2}, \ldots, x_{n}\right) \\
&= f\left(2 x_{1}, 2 x_{2}, \ldots, 2 x_{n}\right)-f\left(x_{1}^{(1)}, x_{2}^{(1)}, \ldots, x_{n}^{(1)}\right)-f\left(x_{1}^{(2)}, x_{2}^{(2)}, \ldots, x_{n}^{(2)}\right) \\
&-2 f\left(x_{1}, x_{2}, \ldots, x_{n}\right)+2 f\left(\frac{x_{1}^{(1)}}{2}, \frac{x_{2}^{(1)}}{2}, \ldots, \frac{x_{n}^{(1)}}{2}\right)+2 f\left(\frac{x_{1}^{(2)}}{2}, \frac{x_{2}^{(2)}}{2}, \ldots, \frac{x_{n}^{(2)}}{2}\right) \\
&+f\left(x_{1}^{(1)}, x_{2}^{(1)}, \ldots, x_{n}^{(1)}\right)-2 f\left(\frac{x_{1}^{(1)}}{2}, \frac{x_{2}^{(1)}}{2}, \ldots, \frac{x_{n}^{(1)}}{2}\right) \\
&+f\left(x_{1}^{(2)}, x_{2}^{(2)}, \ldots, x_{n}^{(2)}\right)-2 f\left(\frac{x_{1}^{(2)}}{2}, \frac{x_{2}^{(2)}}{2}, \ldots, \frac{x_{n}^{(2)}}{2}\right) \\
&= D f\left(x_{1}^{(1)}, x_{1}^{(2)}, x_{2}^{(1)}, x_{2}^{(2)}, \ldots, x_{n}^{(1)}, x_{n}^{(2)}\right)-2 D f\left(\frac{x_{1}^{(1)}}{2}, \frac{x_{1}^{(2)}}{2}, \frac{x_{2}^{(1)}}{2}, \frac{x_{2}^{(2)}}{2}, \ldots, \frac{x_{n}^{(1)}}{2}, \frac{x_{n}^{(2)}}{2}\right) \\
&+D f\left(\frac{x_{1}^{(1)}}{2}, \frac{x_{1}^{(1)}}{2}, \frac{x_{2}^{(1)}}{2}, \frac{x_{2}^{(1)}}{2}, \ldots, \frac{x_{n}^{(1)}}{2}, \frac{x_{n}^{(1)}}{2}\right) \\
&+D f\left(\frac{x_{1}^{(2)}}{2}, \frac{x_{1}^{(2)}}{2}, \frac{x_{2}^{(2)}}{2}, \frac{x_{2}^{(2)}}{2}, \ldots, \frac{x_{n}^{(2)}}{2}, \frac{x_{n}^{(2)}}{2}\right)
\end{aligned}
$$

for all $\left(x_{1}, x_{2}, \ldots, x_{n}\right) \in V^{n} \backslash\{(0,0, \ldots, 0)\}$, we have

$$
\begin{aligned}
&\left\|f\left(x_{1}, x_{2}, \ldots, x_{n}\right)-\frac{f\left(2 x_{1}, 2 x_{2}, \ldots, 2 x_{n}\right)}{2}\right\| \\
& \leq\left\|D f\left(x_{1}^{(1)}, x_{1}^{(2)}, x_{2}^{(1)}, x_{2}^{(2)}, \ldots, x_{n}^{(1)}, x_{n}^{(2)}\right)\right\|+2\left\|D f\left(\frac{x_{1}^{(1)}}{2}, \frac{x_{1}^{(2)}}{2}, \frac{x_{2}^{(1)}}{2}, \frac{x_{2}^{(2)}}{2}, \ldots, \frac{x_{n}^{(1)}}{2}, \frac{x_{n}^{(2)}}{2}\right)\right\| \\
&+\left\|D f\left(\frac{x_{1}^{(1)}}{2}, \frac{x_{1}^{(1)}}{2}, \frac{x_{2}^{(1)}}{2}, \frac{x_{2}^{(1)}}{2}, \ldots, \frac{x_{n}^{(1)}}{2}, \frac{x_{n}^{(1)}}{2}\right)\right\| \\
&+\left\|D f\left(\frac{x_{1}^{(2)}}{2}, \frac{x_{1}^{(2)}}{2}, \frac{x_{2}^{(2)}}{2}, \frac{x_{2}^{(2)}}{2}, \ldots, \frac{x_{n}^{(2)}}{2}, \frac{x_{n}^{(2)}}{2}\right)\right\| \\
& \leq \frac{1}{2} \mu\left(x_{1}, x_{2}, \ldots, x_{n}\right)
\end{aligned}
$$


for all $\left(x_{1}, x_{2}, \ldots, x_{n}\right) \in V^{n} \backslash\{(0,0, \ldots, 0)\}$. From the above inequality, we get the (following4 palces) inequality

$$
\begin{aligned}
\| \frac{f\left(2^{m} x_{1}, \ldots, 2^{m} x_{n}\right)}{2^{m}} & -\frac{f\left(2^{m+m^{\prime}} x_{1}, \ldots, 2^{m+m^{\prime}} x_{n}\right)}{2^{m+m^{\prime}}} \| \\
& \leq \sum_{k=m}^{m+m^{\prime}-1}\left\|\frac{f\left(2^{k} x_{1}, \ldots, 2^{k} x_{n}\right)}{2^{k}}-\frac{f\left(2^{k+1} x_{1}, \ldots, 2^{k+1} x_{n}\right)}{2^{k+1}}\right\| \\
& \leq \sum_{k=m}^{m+m^{\prime}-1} \frac{\mu\left(2^{k} x_{1}, 2^{k} x_{2}, \ldots, 2^{k} x_{n}\right)}{2^{k+1}}
\end{aligned}
$$

for all $\left(x_{1}, x_{2}, \ldots, x_{n}\right) \in V^{n} \backslash\{(0,0, \ldots, 0)\}$ and all positive integers $m, m^{\prime}$. Thus, the sequence $\left\{\frac{f\left(2^{n} x_{1}, \ldots, 2^{n} x_{n}\right)}{2^{n}}\right\}_{m \in \mathbb{N}}$ is a Cauchy sequence for all $\left(x_{1}, x_{2}, \ldots, x_{n}\right) \in V^{n} \backslash\{(0,0, \ldots, 0)\}$. Since $Y$ is a real Banach space and $\lim _{m \rightarrow \infty} \frac{f\left(2^{m} 0,2^{m} 0, \ldots, 2^{m} 0\right)}{2^{m}}=0$, we can define a mapping $F: V^{n} \rightarrow Y$ by

$$
F\left(x_{1}, x_{2}, \ldots, x_{n}\right)=\lim _{m \rightarrow \infty} \frac{f\left(2^{m} x_{1}, 2^{m} x_{2}, \ldots, 2^{m} x_{n}\right)}{2^{m}}
$$

for all $x_{1}, x_{2}, \ldots, x_{n} \in V$. By putting $m=0$ and by letting $m^{\prime} \rightarrow \infty$ in the inequalities (10), we can obtain the inequalities (8) for all $\left(x_{1}, x_{2}, \ldots, x_{n}\right) \in V^{n} \backslash\{(0,0, \ldots, 0)\}$.

From the inequality (6), we can obtain

$$
\left\|\frac{D f\left(2^{m} x_{1}, 2^{m} y_{1}, 2^{m} x_{2}, 2^{m} y_{2}, \ldots, 2^{m} x_{n}, 2^{m} y_{n}\right)}{2^{m}}\right\| \leq \frac{\varphi\left(2^{m} x_{1}, 2^{m} y_{1}, 2^{m} x_{2}, \ldots, 2^{m} x_{n}, 2^{m} y_{n}\right)}{2^{m}}
$$

for all $x_{1}, y_{1}, x_{2}, y_{2}, \ldots, x_{n}, y_{n} \in V \backslash\{0\}$. Since the right-hand side in the above equality tends to zero as $m \rightarrow \infty$, and the equality

$$
D F\left(x_{1}, y_{1}, x_{2}, y_{2}, \ldots, x_{n}, y_{n}\right)=\lim _{m \rightarrow \infty} \frac{D f\left(2^{m} x_{1}, 2^{m} y_{1}, 2^{m} x_{2}, 2^{m} y_{2}, \ldots, 2^{m} x_{n}, 2^{m} y_{n}\right)}{2^{m}}
$$

holds, then $F$ satisfies the equality (7) for all $x_{1}, y_{1}, \ldots, x_{n}, y_{n} \in V \backslash\{0\}$. By Lemma $1, F$ satisfies the equality (3) for all $x_{1}, y_{1}, x_{2}, y_{2}, \ldots, x_{n}, y_{n} \in V$. If $G: V^{n} \rightarrow Y$ is another $n$-variable additive mapping that satisfies (8), then we obtain $G(0,0, \ldots, 0)=0=F(0,0 \ldots, 0)$ and

$$
\begin{aligned}
\| G\left(x_{1}, x_{2}, \ldots, x_{n}\right) & -F\left(x_{1}, x_{2}, \ldots, x_{n}\right) \| \\
\leq & \left\|\frac{G\left(2^{k} x_{1}, 2^{k} x_{2}, \ldots, 2^{k} x_{n}\right)}{2^{k}}-\frac{f\left(2^{k} x_{1}, 2^{k} x_{2}, \ldots, 2^{k} x_{n}\right)}{2^{k}}\right\| \\
& +\left\|\frac{f\left(2^{k} x_{1}, 2^{k} x_{2}, \ldots, 2^{k} x_{n}\right)}{2^{k}}-\frac{F\left(2^{k} x_{1}, 2^{k} x_{2}, \ldots, 2^{k} x_{n}\right)}{2^{k}}\right\| \\
\leq & \sum_{m=k}^{\infty} \frac{\mu\left(2^{m} x_{1}, 2^{m} x_{2}, \ldots, 2^{m} x_{n}\right)}{2^{m}}
\end{aligned}
$$

for all $\left(x_{1}, x_{2}, \ldots, x_{n}\right) \in V^{n} \backslash\{(0,0, \ldots, 0)\}$ and all $k \in \mathbb{N}$. Since $\sum_{m=k}^{\infty} \frac{\mu\left(2^{m} x_{1}, 2^{m} x_{2}, \ldots, 2^{m} x_{n}\right)}{2^{m}} \rightarrow 0$ as $k \rightarrow \infty$, we have $G\left(x_{1}, x_{2}, \ldots, x_{n}\right)=F\left(x_{1}, x_{2}, \ldots, x_{n}\right)$ for all $x_{1}, x_{2}, \ldots, x_{n} \in V$. Hence, the mapping $F$ is the unique $n$-variable additive mapping, as desired.

The condition $x_{1}, y_{1}, x_{2}, y_{2}, \ldots, x_{n}, y_{n} \in V \backslash\{0\}$ used in the inequality (6) differs from the condition $\left(x_{1}, x_{2}, \ldots, x_{n}\right) \neq(0,0, \ldots, 0)$ and $\left(y_{1}, y_{2}, \ldots, y_{n}\right) \neq(0,0, \ldots, 0)$ handled by the other authors. If the function $\mathrm{f}$ satisfies the inequality (3.2) for all $\left(x_{1}, x_{2}, \ldots, x_{n}\right) \neq(0,0, \ldots, 0)$ and $\left(y_{1}, y_{2}, \ldots, y_{n}\right) \neq$ 
$(0,0, \ldots, 0)$, then the function $\mathrm{f}$ satisfies the inequality (3.2) for all $x_{1}, y_{1}, x_{2}, y_{2}, \ldots, x_{n}, y_{n} \in V \backslash\{0\}$. Therefore, the condition $x_{1}, y_{1}, x_{2}, y_{2}, \ldots, x_{n}, y_{n} \in V \backslash\{0\}$ used in the inequality (3.2) in this paper is a generalization of the conditions used in the inequality (3.2) in the well-known pre-results $([10,11])$. This condition will apply until Corollary 1.

Theorem 4. Suppose that $f: V^{n} \rightarrow Y$ is a mapping for which there exists a function $\varphi:(V \backslash\{0\})^{2 n} \rightarrow[0, \infty)$ that satisfies

$$
\sum_{i=0}^{\infty} 2^{i} \varphi\left(\frac{x_{1}}{2^{i}}, \frac{y_{1}}{2^{i}}, \frac{x_{2}}{2^{i}}, \frac{y_{2}}{2^{i}}, \ldots, \frac{x_{n}}{2^{i}}, \frac{y_{n}}{2^{i}}\right)<\infty,
$$

and (6) for all $x_{1}, y_{1}, x_{2}, y_{2}, \ldots, x_{n}, y_{n} \in V \backslash\{0\}$. Then, there exists a unique mapping $F: V^{n} \rightarrow Y$ that satisfies (7) for all $x_{1}, y_{1}, x_{2}, y_{2}, \ldots, x_{n}, y_{n} \in V$ and

$$
\left\|f\left(x_{1}, x_{2}, \ldots, x_{n}\right)-F\left(x_{1}, x_{2}, \ldots, x_{n}\right)\right\| \leq \sum_{m=0}^{\infty} 2^{m} \mu\left(\frac{x_{1}}{2^{m+1}}, \frac{x_{2}}{2^{m+1}}, \ldots, \frac{x_{n}}{2^{m+1}}\right)
$$

for all $\left(x_{1}, x_{2}, \ldots, x_{n}\right) \in V^{n} \backslash\{(0,0, \ldots, 0)\}$, where the function $\mu: V^{n} \rightarrow \mathbb{R}$ is defined as Theorem 3 .

Proof. By choosing a fixed element $x \in V \backslash\{0\}$, we can obtain

$$
\begin{aligned}
\|f(0,0, \ldots, 0)\|= & \| 2 D f\left(\frac{x}{2^{m}}, \frac{-x}{2^{m}}, \ldots, \frac{x}{2^{m}}, \frac{-x}{2^{m}}\right)-D f\left(\frac{x}{2^{m-1}}, \frac{-x}{2^{m-1}}, \ldots, \frac{x}{2^{m-1}}, \frac{-x}{2^{m-1}}\right) \\
& -D f\left(\frac{x}{2^{m}}, \frac{x}{2^{m}}, \ldots, \frac{x}{2^{m}}, \frac{x}{2^{m}}\right)-D f\left(\frac{-x}{2^{m}}, \frac{-x}{2^{m}}, \ldots, \frac{-x}{2^{m}}, \frac{-x}{2^{m}}\right) \| \\
\leq & 2 \varphi\left(\frac{x}{2^{m}}, \frac{-x}{2^{m}}, \ldots, \frac{x}{2^{m}}, \frac{-x}{2^{m}}\right)+\varphi\left(\frac{x}{2^{m-1}}, \frac{-x}{2^{m-1}}, \ldots, \frac{x}{2^{m-1}}, \frac{-x}{2^{m-1}}\right) \\
& +\varphi\left(\frac{x}{2^{m}}, \frac{x}{2^{m}}, \ldots, \frac{x}{2^{m}}, \frac{x}{2^{m}}\right)+\varphi\left(\frac{-x}{2^{m}}, \frac{-x}{2^{m}}, \ldots, \frac{-x}{2^{m}}, \frac{-x}{2^{m}}\right) \\
\rightarrow & 0 \text { as } m \rightarrow \infty,
\end{aligned}
$$

so $f(0,0, \ldots, 0)=0$. Since the equality (9) holds for all $\left(x_{1}, x_{2}, \ldots, x_{n}\right) \in V \backslash\{(0,0, \ldots, 0)\}$, the inequality (6) implies the inequality

$$
\left\|f\left(x_{1}, x_{2}, \ldots, x_{n}\right)-2 f\left(\frac{x_{1}}{2}, \frac{x_{2}}{2}, \ldots, \frac{x_{n}}{2}\right)\right\| \leq \mu\left(\frac{x_{1}}{2}, \frac{x_{2}}{2}, \ldots, \frac{x_{n}}{2}\right)
$$

for all $\left(x_{1}, x_{2}, \ldots, x_{n}\right) \in V^{n} \backslash\{(0,0, \ldots, 0)\}$. From the above inequality, we can also obtain the inequality

$$
\begin{gathered}
\left\|2^{m} f\left(\frac{x_{1}}{2^{m}}, \frac{x_{2}}{2^{m}}, \ldots, \frac{x_{n}}{2^{m}}\right)-2^{m+m^{\prime}} f\left(\frac{x_{1}}{2^{m+m^{\prime}}}, \frac{x_{2}}{2^{m+m^{\prime}}}, \ldots, \frac{x_{n}}{2^{m+m^{\prime}}}\right)\right\| \\
\leq \sum_{k=m}^{m+m^{\prime}-1} 2^{k} \mu\left(\frac{x_{1}}{2^{k+1}}, \frac{x_{2}}{2^{k+1}}, \ldots, \frac{x_{n}}{2^{k+1}}\right)
\end{gathered}
$$

for all $\left(x_{1}, x_{2}, \ldots, x_{n}\right) \in V^{n} \backslash\{(0,0, \ldots, 0)\}$ and all positive integers $m, m^{\prime}$. Thus, the sequences $\left\{2^{m} f\left(\frac{x_{1}}{2^{m}}, \ldots, \frac{x_{n}}{2^{m}}\right)\right\}_{m \in \mathbb{N}}$ is a Cauchy sequence for all $\left(x_{1}, \ldots, x_{n}\right) \in V^{n} \backslash\{(0, \ldots, 0)\}$. Since $f(0,0, \ldots, 0)=0$ and $Y$ is a real Banach space, we can define a mapping $F: V^{n} \rightarrow Y$ by

$$
F\left(x_{1}, x_{2}, \ldots, x_{n}\right)=\lim _{m \rightarrow \infty} 2^{m} f\left(\frac{x_{1}}{2^{m}}, \frac{x_{2}}{2^{m}}, \ldots, \frac{x_{n}}{2^{m}}\right)
$$

for all $x_{1}, x_{2}, \ldots, x_{n} \in V$. By putting $m=0$ and by letting $m^{\prime} \rightarrow \infty$ in the inequality (13), we can obtain the inequality (12) for all $\left(x_{1}, x_{2}, \ldots, x_{n}\right) \in V^{n} \backslash\{(0,0, \ldots, 0)\}$. 
From the inequality (6), we get

$$
\left\|2^{m} D f\left(\frac{x_{1}}{2^{m}}, \frac{y_{1}}{2^{m}}, \frac{x_{2}}{2^{m}}, \frac{y_{2}}{2^{m}}, \ldots, \frac{x_{n}}{2^{m}}, \frac{y_{n}}{2^{m}}\right)\right\| \leq 2^{m} \varphi\left(\frac{x_{1}}{2^{m}}, \frac{y_{1}}{2^{m}}, \frac{x_{2}}{2^{m}}, \frac{y_{2}}{2^{m}}, \ldots, \frac{x_{n}}{2^{m}}, \frac{y_{n}}{2^{m}}\right)
$$

for all $x_{1}, y_{1}, x_{2}, y_{2}, \ldots, x_{n}, y_{n} \in V \backslash\{0\}$. Since the right-hand side in the above equality tends to zero as $m \rightarrow \infty$, then $F$ satisfies the equality (7) for all $x_{1}, y_{1}, x_{2}, y_{2}, \ldots, x_{n}, y_{n} \in V \backslash\{0\}$. By Lemma $1, F$ satisfies the equality (3) for all $x_{1}, y_{1}, x_{2}, y_{2}, \ldots, x_{n}, y_{n} \in V$. If $G: V^{n} \rightarrow Y$ is another $n$-variable additive mapping satisfying (12), then we obtain $G(0,0, \ldots, 0)=0=F(0,0, \ldots, 0)$ and

$$
\begin{aligned}
\| G\left(x_{1}, x_{2}, \ldots, x_{n}\right) & -F\left(x_{1}, x_{2}, \ldots, x_{n}\right) \| \\
\leq & \left\|2^{k} G\left(\frac{x_{1}}{2^{k}}, \frac{x_{2}}{2^{k}}, \ldots, \frac{x_{n}}{2^{k}}\right)-2^{k} f\left(\frac{x_{1}}{2^{k}}, \frac{x_{2}}{2^{k}}, \ldots, \frac{x_{n}}{2^{k}}\right)\right\| \\
& +\left\|2^{k} f\left(\frac{x_{1}}{2^{k}}, \frac{x_{2}}{2^{k}}, \ldots, \frac{x_{n}}{2^{k}}\right)-2^{k} F\left(\frac{x_{1}}{2^{k}}, \frac{x_{2}}{2^{k}}, \ldots, \frac{x_{n}}{2^{k}}\right)\right\| \\
\leq & \sum_{m=k}^{\infty} 2^{m} \mu\left(\frac{x_{1}}{2^{m+1}}, \frac{x_{2}}{2^{m+1}}, \ldots, \frac{x_{n}}{2^{m+1}}\right) \\
\rightarrow & 0 \text { as } k \rightarrow \infty
\end{aligned}
$$

for all $\left(x_{1}, x_{2}, \ldots, x_{n}\right) \in V^{n} \backslash\{(0,0, \ldots, 0)\}$. Hence, the mapping $F$ is the unique $n$-variable additive mapping, as desired.

The following corollary follows from Theorems 3 and 4.

Corollary 1. Let $(X,\||\|\mid\|)$ be a normed space, $\theta>0$, and let $p$ be a real number with $p \neq 1$. Suppose that $f: X^{n} \rightarrow Y$ is a mapping that satisfies

$$
\left\|D f\left(x_{1}, y_{1}, x_{2}, y_{2}, \ldots, x_{n}, y_{n}\right)\right\| \leq \theta\left(\left.\left\||| x_{1}||^{p}+\right\||| y_{1}||\right|^{p}+\left\|\left|x_{2}\right|||^{p}+\ldots+\right\||| x_{n}||^{p}+\|\left|y_{n}\right|||^{p}\right)
$$

for all $x_{1}, y_{1}, x_{2}, y_{2}, \ldots, x_{n}, y_{n} \in X \backslash\{0\}$. Then, there exists a unique n-variable additive mapping $F: X^{n} \rightarrow Y$, such that

$$
\left\|f\left(x_{1}, x_{2}, \ldots, x_{n}\right)-F\left(x_{1}, x_{2}, \ldots, x_{n}\right)\right\| \leq \frac{4\left(2^{p}+4\right) n \theta}{2^{p}\left|2-2^{p}\right|} \max _{x_{i} \neq 0}\left\{\left\||| x_{i} \mid\right\|^{p}: 1 \leq i \leq n\right\}
$$

for all $\left(x_{1}, x_{2}, \ldots, x_{n}\right) \in X^{n} \backslash\{(0,0, \ldots, 0)\}$.

Proof. Put $\varphi\left(x_{1}, y_{1}, x_{2}, y_{2}, \ldots, x_{n}, y_{n}\right):=\theta\left(||\left|x_{1}\right|||^{p}+\left.\left\|\left.|| y_{1}||\right|^{p}+\right\||| x_{2}||\right|^{p}+\left.\left\|\left.|| y_{2}||\right|^{p}+\ldots+\right\||| x_{n}||\right|^{p}+\right.$ $\left.\left\|\mid y_{n}\right\|^{p}\right)$ for all $x_{1}, y_{1}, x_{2}, y_{2}, \ldots, x_{n}, y_{n} \in X \backslash\{0\}$, then $\left\|\left|x_{i}^{(1)}\|\|,\left\|\mid x_{i}^{(2)}\right\| \| \leq \max _{x_{i} \neq 0}\left\{\left\||| x_{i}|\||^{p}: 1 \leq i \leq\right.\right.\right.\right.$ $n\}$ for all $i$ from (4). Hence, due to $\mu$ of Theorems 3 and 4 , we obtain that

$$
\begin{aligned}
& \mu\left(x_{1}, x_{2}, \ldots, x_{n}\right) \\
& =\varphi\left(x_{1}^{(1)}, x_{1}^{(2)}, x_{2}^{(1)}, x_{2}^{(2)}, \ldots, x_{n}^{(1)}, x_{n}^{(2)}\right)+2 \varphi\left(\frac{x_{1}^{(1)}}{2}, \frac{x_{1}^{(2)}}{2}, \frac{x_{2}^{(1)}}{2}, \frac{x_{2}^{(2)}}{2}, \ldots, \frac{x_{n}^{(1)}}{2}, \frac{x_{n}^{(2)}}{2}\right) \\
& \quad+\varphi\left(\frac{x_{1}^{(1)}}{2}, \frac{x_{1}^{(1)}}{2}, \frac{x_{2}^{(1)}}{2}, \frac{x_{2}^{(1)}}{2}, \ldots, \frac{x_{n}^{(1)}}{2}, \frac{x_{n}^{(1)}}{2}\right)+\varphi\left(\frac{x_{1}^{(2)}}{2}, \frac{x_{1}^{(2)}}{2}, \frac{x_{2}^{(2)}}{2}, \frac{x_{2}^{(2)}}{2}, \ldots, \frac{x_{n}^{(2)}}{2}, \frac{x_{n}^{(2)}}{2}\right) \\
& \leq\left(2 n+\frac{8 n}{2^{p}}\right) \max _{x_{i} \neq 0}\left\{\left\||| x_{i} \mid\right\|^{p}: 1 \leq i \leq n\right\}
\end{aligned}
$$

for all $\left(x_{1}, x_{2}, \ldots, x_{n}\right) \in X^{n} \backslash\{(0,0, \ldots, 0)\}$. Therefore, the inequality (15) can be obtained easily from (8) and (12) in Theorems 3 and 4. 
The following theorem for the hyperstability of $n$-variable additive functional equation follows from Corollary 1.

Theorem 5. Let $(X,|||\cdot|||)$ be a normed space and $p$ be a real number with $p<0$. Suppose that $f: X^{n} \rightarrow Y$ is a mapping that satisfies (14) for all $x_{1}, y_{1}, x_{2}, y_{2}, \ldots, x_{n}, y_{n} \in X \backslash\{0\}$. Then, $f$ is an $n$-variable additive mapping itself.

Proof. By Corollary 1, there exists a unique $n$-variable additive mapping $F: X^{n} \rightarrow Y$, such that (15) for all $x_{1}, x_{2}, \ldots, x_{n} \in X^{n} \backslash\{(0,0, \ldots, 0)\}$ and $D F\left(x_{1}, y_{1}, x_{2}, y_{2}, \ldots, x_{n}, y_{n}\right)=0$ for all $x_{1}, y_{1}, x_{2}, y_{2}, \ldots, x_{n}, y_{n} \in X$. and let

For a given $\left(x_{1}, x_{2}, \ldots, x_{n}\right) \neq(0,0, \ldots, 0)$, let $x^{\prime} \neq 0$ be a nonzero fixed element in $\left\{x_{1}, x_{2}, \ldots, x_{n}\right\}$,

$$
\begin{array}{ll}
x_{i}^{(3)}=(m+1) x_{i}, x_{i}^{(4)}=-m x_{i} & \text { when } x_{i} \neq 0, \\
x_{i}^{(3)}=m x^{\prime}, x_{i}^{(4)}=-m x^{\prime} & \text { when } x_{i}=0 .
\end{array}
$$

Then, we can easily show that $\left\|\left|x_{i}^{(3)}\right|\right\|,\left|\left\|x_{i}^{(4)} \mid\right\| \leq m^{p} \max _{x_{i} \neq 0}\left\{\left\|\left|x_{i} \|\right|^{p}: 1 \leq i \leq n\right\}\right.\right.$ for all $i$ from (4). If $\left(x_{1}, x_{2}, \ldots, x_{n}\right) \in X \backslash\{(0,0, \ldots, 0)\}$, then the equality $f\left(x_{1}, x_{2}, \ldots, x_{n}\right)=F\left(x_{1}, x_{2}, \ldots, x_{n}\right)$ follows from the inequalities

$$
\begin{aligned}
&\left\|f\left(x_{1}, x_{2}, \ldots, x_{n}\right)-F\left(x_{1}, x_{2}, \ldots, x_{n}\right)\right\| \\
&= \| D f\left(x_{1}^{(3)}, x_{1}^{(4)}, x_{2}^{(3)}, x_{2}^{(4)}, \ldots, x_{n}^{(3)}, x_{n}^{(4)}\right)-D F\left(x_{1}^{(3)}, x_{1}^{(4)}, x_{2}^{(3)}, x_{2}^{(4)}, \ldots, x_{n}^{(3)}, x_{n}^{(4)}\right) \\
&+f\left(x_{1}^{(3)}, x_{2}^{(3)}, \ldots,\left(x_{n}^{(3)}\right)+f\left(x_{1}^{(4)}, x_{2}^{(4)}, \ldots, x_{n}^{(4)}\right)\right. \\
& \quad-F\left(x_{1}^{(3)}, x_{2}^{(3)}, \ldots,\left(x_{n}^{(3)}\right)-F\left(x_{1}^{(4)}, x_{2}^{(4)}, \ldots, x_{n}^{(4)}\right) \|\right. \\
& \leq m^{p} \cdot 2 n \theta \max _{x_{i} \neq 0}\left\{\left\|\mid x_{i}\right\| \|^{p}: 1 \leq i \leq n\right\}+\left\|f\left(x_{1}^{(3)}, x_{2}^{(3)}, \ldots, x_{n}^{(3)}\right)-F\left(x_{1}^{(3)}, x_{2}^{(3)}, \ldots, x_{n}^{(3)}\right)\right\| \\
&+\left\|f\left(x_{1}^{(4)}, x_{2}^{(4)}, \ldots, x_{n}^{(4)}\right)-F\left(x_{1}^{(4)}, x_{2}^{(4)}, \ldots, x_{n}^{(4)}\right)\right\| \\
& \leq m^{p}\left(1+\frac{4\left(2^{p}+4\right)}{2^{p}\left|2-2^{p}\right|}\right) 2 n \theta \max _{x_{i} \neq 0}\left\{\mid\left\|x_{i}\right\| \|^{p}: 1 \leq i \leq n\right\}
\end{aligned}
$$

as $m \rightarrow \infty$. For $\left(x_{1}, x_{2}, \ldots, x_{n}\right)=(0,0, \ldots, 0)$, if we choose a fixed element of $x \in X \backslash\{0\}$, then the equality $f 0,0, \ldots, 0)=F 0,0, \ldots, 0)$ follows from the inequalities

$$
\begin{aligned}
&\|f(0,0, \ldots, 0)-F(0,0, \ldots, 0)\| \\
&= \| D f(m x,-m x, m x,-m x, \ldots, m x,-m x)-D F(m x,-m x, m x, \ldots, m x,-m x) \\
&+f(m x, m x, \ldots, m x)+f(-m x,-m x, \ldots,-m x) \\
& \quad-F(m x, m x, \ldots, m x)-F(-m x,-m x, \ldots,-m x) \| \\
& \leq m^{p} \cdot 2 n \theta\|x\|^{p}+\|f(m x, m x, \ldots, m x)-F(m x, m x, \ldots, m x)\| \\
&+\|f(-m x,-m x, \ldots,-m x)-F(-m x,-m x, \ldots,-m x)\| \\
& \leq m^{p}\left(1+\frac{4\left(2^{p}+4\right)}{2^{p}\left|2-2^{p}\right|}\right) 2 n \theta\|\| x\|\|^{p}
\end{aligned}
$$

as $m \rightarrow \infty$. Therefore, $f$ is an $n$-variable additive mapping itself.

The following example follows from Theorem 5 . 
Example 1. Let $(\mathbb{R},|\cdot|)$ be a normed space with absolute value $|\cdot|,\left(\mathbb{R}^{l},\|\cdot\|\right)$ be a Banach space with Euclid norm $\|\cdot\|$, and $p<0$ be a real number. Suppose that $f: \mathbb{R}^{n} \rightarrow \mathbb{R}^{l}$ is a continuous mapping such that

$$
\left\|D f\left(x_{1}, y_{1}, x_{2}, y_{2}, \ldots, x_{n}, y_{n}\right)\right\| \leq \theta\left(\left|x_{1}\right|^{p}+\left|y_{1}\right|^{p}+\left|x_{2}\right|^{p}+\left|y_{2}\right|^{p}+\ldots+\left|x_{n}\right|^{p}+\left|y_{n}\right|^{p}\right)
$$

for all $x_{1}, y_{1}, x_{2}, y_{2}, \ldots, x_{n}, y_{n} \in \mathbb{R} \backslash\{0\}$. Then, the mapping $f: \mathbb{R}^{n} \rightarrow \mathbb{R}^{l}$ given by

$$
f\left(x_{1}, x_{2}, \ldots, x_{n}\right)=\left(\sum_{i=1}^{n} a_{1 i} x_{i}, \sum_{i=1}^{n} a_{2 i} x_{i}, \ldots, \sum_{i=1}^{n} a_{l i} x_{i}\right),
$$

where $a_{1 i}, a_{2 i}, \ldots, a_{l i}$ are real constants, indicates that

$$
\begin{gathered}
f(1,0,0, \ldots, 0)=\left(a_{11}, a_{21}, \ldots, a_{l 1}\right), \\
f(0,1,0, \ldots, 0)=\left(a_{12}, a_{22}, \ldots, a_{l 2}\right), \\
\vdots \quad \vdots \\
f(0, \ldots, 0,0,1)=\left(a_{1 n}, a_{2 n}, \ldots, a_{l n}\right) .
\end{gathered}
$$

Proof. Since $f: \mathbb{R}^{n} \rightarrow \mathbb{R}^{l}$ is a continuous $n$-variable additive mapping by Theorem 5 , then the function $f: \mathbb{R}^{n} \rightarrow \mathbb{R}^{l}$ is given by (16).

In the following theorems, we replace the domain $(V \backslash\{0\})^{2 n}$ of $\varphi$ and $D f$ in Theorems 3 and 4 with $V^{2 n}$. Then, we can improve the result inequality (8).

Theorem 6. Suppose that $f: V^{n} \rightarrow Y$ is a mapping for which there exists a function $\varphi: V^{2 n} \rightarrow[0, \infty)$ satisfying (5) and (6) for all $x_{1}, y_{1}, x_{2}, y_{2}, \ldots, x_{n}, y_{n} \in V$. Then, there exists a unique mapping $F: V^{n} \rightarrow Y$, such that (7) for all $x_{1}, y_{1}, x_{2}, y_{2}, \ldots, x_{n}, y_{n} \in V$ and

$$
\left\|f\left(x_{1}, x_{2}, \ldots, x_{n}\right)-F\left(x_{1}, x_{2}, \ldots, x_{n}\right)\right\| \leq \sum_{m=0}^{\infty} \frac{\varphi\left(2^{m} x_{1}, 2^{m} x_{1}, 2^{m} x_{2}, \ldots, 2^{m} x_{n}, 2^{m} x_{n}\right)}{2^{m+1}}
$$

for all $x_{1}, x_{2}, \ldots, x_{n} \in V$.

Proof. The equality

$$
f\left(2 x_{1}, 2 x_{2}, \ldots, 2 x_{n}\right)-2 f\left(x_{1}, x_{2}, \ldots, x_{n}\right)=D f\left(x_{1}, x_{1}, x_{2}, x_{2}, \ldots, x_{n}, x_{n}\right)
$$

for all $x_{1}, x_{2}, \ldots, x_{n} \in V$ and the inequality (6) imply that the inequality

$$
\left\|f\left(x_{1}, x_{2}, \ldots, x_{n}\right)-\frac{f\left(2 x_{1}, 2 x_{2}, \ldots, 2 x_{n}\right)}{2}\right\| \leq \frac{1}{2} \varphi\left(x_{1}, x_{1}, x_{2}, x_{2}, \ldots, x_{n}, x_{n}\right)
$$

for all $x_{1}, x_{2}, \ldots, x_{n} \in V$. From the above inequality, we can derive the inequalities

$$
\begin{aligned}
\| \frac{f\left(2^{m} x_{1}, \ldots, 2^{m} x_{n}\right)}{2^{m}} & -\frac{f\left(2^{m+m^{\prime}} x_{1}, \ldots, 2^{m+m^{\prime}} x_{n}\right)}{2^{m+m^{\prime}}} \| \\
& \leq \sum_{k=m}^{m+m^{\prime}-1} \frac{\varphi\left(2^{k} x_{1}, 2^{k} x_{1}, 2^{k} x_{2}, 2^{k} x_{2}, \ldots, 2^{k} x_{n}, 2^{k} x_{n}\right)}{2^{k+1}}
\end{aligned}
$$

for all $x_{1}, x_{2}, \ldots, x_{n} \in V$ and all positive integers $m, m^{\prime}$. The remainder of the proof of this theorem developed after inequality (19) is omitted because it is similar to that of Theorem 3. 
Theorem 7. Suppose that $f: V^{n} \rightarrow Y$ is a mapping for which there exists a function $\varphi: V^{2 n} \rightarrow[0, \infty)$ satisfying (11) and (6) for all $x_{1}, y_{1}, x_{2}, y_{2}, \ldots, x_{n}, y_{n} \in V$. Then, there exists a unique mapping $F: V^{n} \rightarrow Y$ that satisfies (7) for all $x_{1}, y_{1}, x_{2}, y_{2}, \ldots, x_{n}, y_{n} \in V$ and

$$
\left\|f\left(x_{1}, \ldots, x_{n}\right)-F\left(x_{1}, \ldots, x_{n}\right)\right\| \leq \sum_{m=0}^{\infty} 2^{m} \varphi\left(\frac{x_{1}}{2^{m+1}}, \frac{x_{1}}{2^{m+1}}, \frac{x_{2}}{2^{m+1}}, \ldots, \frac{x_{n}}{2^{m+1}}, \frac{x_{n}}{2^{m+1}}\right)
$$

for all $x_{1}, x_{2}, \ldots, x_{n} \in V$.

Proof. The equality (18) for all $x_{1}, x_{2}, \ldots, x_{n} \in V$ and the inequality (6) imply that the inequality

$$
\left\|f\left(x_{1}, x_{2}, \ldots, x_{n}\right)-2 f\left(\frac{x_{1}}{2}, \frac{x_{2}}{2}, \ldots, \frac{x_{n}}{2}\right)\right\| \leq \varphi\left(\frac{x_{1}}{2}, \frac{x_{1}}{2}, \frac{x_{2}}{2}, \ldots, \frac{x_{n}}{2}, \frac{x_{n}}{2}\right)
$$

for all $x_{1}, x_{2}, \ldots, x_{n} \in V$. From the above inequality, we can derive the inequality

$$
\begin{array}{r}
\left\|2^{m} f\left(\frac{x_{1}}{2^{m}}, \frac{x_{2}}{2^{m}}, \ldots, \frac{x_{n}}{2^{m}}\right)-2^{m+m^{\prime}} f\left(\frac{x_{1}}{2^{m+m^{\prime}}}, \frac{x_{2}}{2^{m+m^{\prime}}}, \ldots, \frac{x_{n}}{2^{m+m^{\prime}}}\right)\right\| \\
\leq \sum_{k=m}^{m+m^{\prime}-1} 2^{k} \varphi\left(\frac{x_{1}}{2^{k+1}}, \frac{x_{1}}{2^{k+1}}, \frac{x_{2}}{2^{k+1}}, \ldots, \frac{x_{n}}{2^{k}}, \frac{x_{n}}{2^{k}}\right)
\end{array}
$$

for all $x_{1}, x_{2}, \ldots, x_{n} \in V$ and all positive integers $m, m^{\prime}$. The remainder of the proof of this theorem developed after inequality (21) is omitted because it is similar to that of Theorem 4.

The following corollary follows from Theorems 6 and 7.

Corollary 2. Let $(X, \||| \cdot||)$ be a normed space and $p$ be a nonnegative real number with $p \neq 1$. Suppose that $f: X^{n} \rightarrow Y$ is a mapping satisfying (14) for all $x_{1}, y_{1}, x_{2}, y_{2}, \ldots, x_{n}, y_{n} \in X$. Then, there exists a unique $n$-variable additive mapping $F: X^{n} \rightarrow Y$ such that

$$
\left\|f\left(x_{1}, x_{2}, \ldots, x_{n}\right)-F\left(x_{1}, x_{2}, \ldots, x_{n}\right)\right\| \leq \frac{2 \theta}{\left|2-2^{p}\right|}\left(||\left|x_{1}\right|\left\|\left.\right|^{p}+\right\|\left|x_{2}\right|||^{p}+\ldots+\left\||| x_{n}||\right\|^{p}\right)
$$

for all $x_{1}, x_{2}, \ldots, x_{n} \in X$

Proof. By putting $\varphi\left(x_{1}, y_{1}, x_{2}, y_{2}, \ldots, x_{n}, y_{n}\right):=\theta\left(||\left|x_{1}\right|\left|\left\|^{p}+\right\|\right|\left|y_{1}\right|||^{p}+\left\||| x_{2}||\right\|^{p}+\||| y_{2}||||^{p}+\cdots+\right.$ $\left.\left\|\left|x_{n}\left\|^{p}+\right\|\right| y_{n}\right\|^{p}\right)$ for all $x_{1}, y_{1}, x_{2}, y_{2}, \ldots, x_{n}, y_{n} \in X$, then we easily obtain (22) from (17) and (20) of Theorems 6 and 7.

\section{Conclusions}

We obtained two stability results.

Theorems 3 and 4 are the generalized Hyers-Ulam stability for the additive functional Equation (3) on $V^{n}$, which is a generalization for the stability of the Cauchy functional equation in papers of Aoki [3], Rassias [4], Gajda [5], Hyers [2], and Găvruta [7].

Theorems 6 and 7 are the hyperstablity of the additive functional Equation (3) on $V^{n}$, which is a generalization of the Brzdęk's results $[10,11]$ for the Cauchy functional equation.

If the function $\mathrm{f}$ satisfies the inequality (6) for all $\left(x_{1}, x_{2}, \ldots, x_{n}\right) \neq(0,0, \ldots, 0)$ and $\left(y_{1}, y_{2}, \ldots, y_{n}\right) \neq(0,0, \ldots, 0)$, then the function $\mathrm{f}$ satisfies the inequality (6) for all $x_{1}, y_{1}, x_{2}, y_{2}, \ldots, x_{n}, y_{n} \in V \backslash\{0\}$. Therefore, the condition $x_{1}, y_{1}, x_{2}, y_{2}, \ldots, x_{n}, y_{n} \in V \backslash\{0\}$ used in the inequality (3.2) of this paper is a generalization of the conditions used in the inequality (6) in well-known pre-results $([10,11])$. 
Author Contributions: Conceptualization, Y.-H.L. and G.H.K.; Investigation, Y.-H.L. and G.H.K.

Funding: This research received no external funding.

Conflicts of Interest: The authors declare no conflict of interest.

\section{References}

1. Ulam, S.M. Problems in Modern Mathematics; Wiley: New York, NY, USA, 1964.

2. Hyers, D.H. On the stability of the linear functional equation. Proc. Natl. Acad. Sci. USA 1941, 27, $222-224$. [CrossRef] [PubMed]

3. Aoki, T. On the stability of the linear transformation in Banach algebras. J. Math. Soc. Japan 1950, 2, 64-66. [CrossRef]

4. Rassias, T.M. On the stability of the linear mapping in Banach spaces. Proc. Am. Math. Soc. 1978, 72, $297-300$. [CrossRef]

5. Gajda, Z. On stability of additive mappings. Int. J. Math. Math. Sci. 1991, 14, 431-434. [CrossRef]

6. Rassias, T.M.; Semrl, P. On the behavior of mappings which do not satisfy Hyers-Ulam stability. Proc. Am. Math. Soc. 1992, 114, 989-993. [CrossRef]

7. Găvruta, P. A generalization of the Hyers-Ulam-Rassias stability of approximately additive mappings. J. Math. Anal. Appl. 1994, 184, 431-436. [CrossRef]

8. Maksa, G.; Páles, Z. Hyperstability of a class of linear functional equations. Acta Math. Acad. Paedagog. Nyházi 2001, 17, 107-112.

9. Bourgin, D.G. Approximately isometric and multiplicative transformations on continuous function rings. Duke Math. J. 1949, 16, 385-397. [CrossRef]

10. Brzdęk, J. Remarks on hyperstability of the the Cauchy equation. Aequationes Math. 2013, 86, $255-267$. [CrossRef]

11. Brzdęk, J. A hyperstability result for the Cauchy equation. Bull. Aust. Math. Soc. 2014, 89, 33-40. [CrossRef]

(C) 2019 by the authors. Licensee MDPI, Basel, Switzerland. This article is an open access article distributed under the terms and conditions of the Creative Commons Attribution (CC BY) license (http:/ / creativecommons.org/licenses/by/4.0/). 